\title{
Canales web y mediatecas en línea como instrumentos de producción, recuperación y difusión en ciencias de la información
}

\author{
Web channels and online mediathecas as tools for the production, retrieval \\ and dissemination of research in information science
}

\begin{abstract}
Alfonso López YePes (1) (*) y Víctor CÁmARA BADos (2) $\left(^{* *}\right)$
(1) Servicios de Documentación Multimedia, Departamento de Bibliotecomía y Documentación, Facultad de Ciencias de la Información, Universidad Complutense de Madrid, alopez@ccinf.ucm.es. (2) vcamarab@ccinf.ucm.es
\end{abstract}

\begin{abstract}
Resumen
Se describe la estructura de contenidos y funcionamiento del canal web de radio-televisión y mediateca en línea RTVDoc, integrado en el Servicio de Documentación Multimedia (Multidoc) de la Facultad de Ciencias de la Información de la Universidad Complutense de Madrid. Este canal temático de documentación vía internet produce, canaliza y difunde contenidos de forma distribuida y colaborativa. Entendido como plataforma universitaria en línea, en tiempo real y "a la carta", el canal apuesta definitivamente por la creación y publicación para docentes, discentes, investigadores y profesionales de medios de comunicación de una amplia variedad de materiales multimedia, didácticos y de aplicación al ámbito docente y profesional, materializados en las correspondientes hemeroteca, fonoteca, fototeca y videoteca. Se aportan varios ejemplos de recuperación de contenidos relacionados con cine, prensa, publicidad y relaciones públicas, radio y televisión, mencionándose asimismo la integración del canal en ámbitos de estudio e investigación diversos. Se alude, en fin, a las colaboraciones ya establecidas en el marco universidadempresa informativa y a la difusión informativa constante del canal temático y sus perspectivas futuras.
\end{abstract}

Palabras clave: Canal web. Documentación informativa. IPTV. Mediateca en línea. Multimedia bajo demanda. Programación radiofónica. Programación de televisión. Producción multimedia. RTVDoc

\section{RTVDoc: creación, proceso evolutivo, nuevo diseño}

El Departamento de Biblioteconomía y Documentación de la Universidad Complutense de Madrid y su Servicio de Documentación Multimedia se han hecho eco constantemente de la evolución de las nuevas tecnologías y de su influencia, participando de ellas y apostando por su implantación en sus diversos ámbitos de actuación. La tecnología documental, en suma, ha estado presente en todo momento en la evo-

\begin{abstract}
The structure of contents and setup of RTVDoc, the radio-and-television web channel and onlinemediatheca of the Documentation Multimedia Service (Multidoc) of the Faculty of Communication Sciences of the University Complutense of Madrid. This thematic channel produces, canalizes and spreads contents in a distributed and collaborative way. Shaped as an online university platform, real-time and "ondemand" service, the channel is the materialization of the idea of creating and publishing materials for teachers, students, researchers and professionals of mass media. The wide variety of multimedia didactic materials are targeted to the educational and professionals sectors. These contents can be found organized in the newspaper library, music library, photo library and video library categories of the channel. Several examples of contributed contents are presented, relating to cinema, press, advertising and public relations, and radio and television. Finally, the different collaborations established by the university and several companies for the continuous diffusion of the thematic channel are discussed, and future overcomes.
\end{abstract}

Keywords: Radio broadcasting. Television broadcasting. Channel web. Information Science. IPTV. Online mediatheca. On-demand multimedia. Multimedia production. RTVDoc.

lución del departamento, y así lo atestiguan las realizaciones y actuaciones constantes por parte del mismo en forma de publicaciones, cursos y seminarios, participación en congresos y otros eventos, proyectos de investigación, dirección de tesis doctorales, formación semipresencial y virtual, etc. Dicha trayectoria se materializó, como otro eslabón más, durante 2007 , en la propuesta inicial de establecimiento de un canal IPTV, en muy estrecha relación con la red social, y en cuya difusión informativa participó El Profesional de la Información con la publicación 
de un trabajo de uno de los autores de este artículo (López, Sánchez , 2007).

En estos dos últimos años, 2007 y $2008-y$ proliferará todavía más en años sucesivos-, la filosofía de los canales IPTV ha seguido en auge a través de publicaciones diversas y artículos de prensa tanto diaria como periódica: Ya es un hecho contrastado la convergencia de televisión e internet, apoyada en manifestaciones tales como que la televisión tradicional no morirá, sino que se transformará y convivirá con las nuevas plataformas de web tv; o que estamos en un momento de transición hacia un modelo de web tv, que ofrecerá contenidos profesionales; o que los usuarios de internet de EE.UU. vieron un total de 13.500 millones de vídeos online durante octubre de 2008 , un $45 \%$ más que en el mismo mes de 2007; o, en fin, que estamos en la tercera era de la televisión en la que la audiencia cada vez tiene un mayor control sobre los contenidos (Cabezas, Barbosa, Serrano, Zurbano, 2008; Jiménez, 2008).

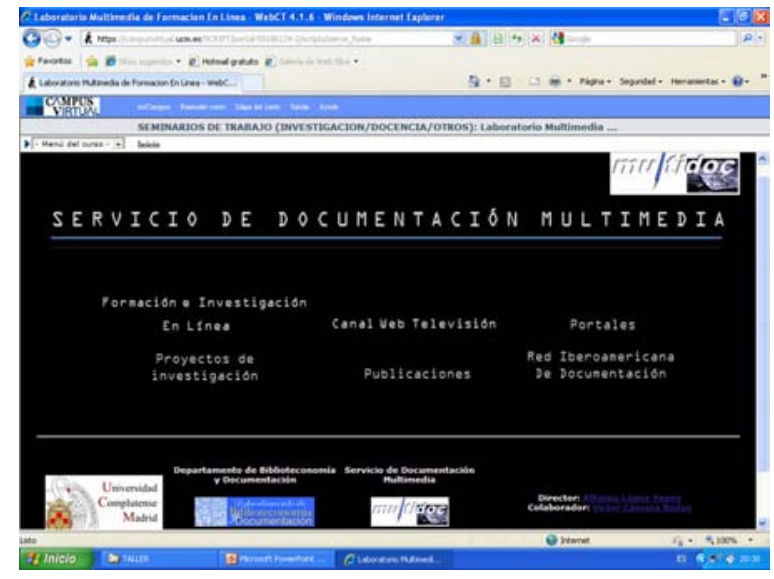

Figura 1

En nuestro ámbito de trabajo, en el que se ha evolucionado hacia todavía una mayor convergencia e integración de medios (multimedia), debe destacarse que tras dos años de su creación, el canal web-mediateca ha sufrido cambios e incluso presenta un nuevo diseño (así como el propio Servicio de Documentación Multimedia, en el que se encuentra integrado) (Figura 1), merced a la incorporación al mismo de nuevas posibilidades de producción y difusión de contenidos, materializados incluso en la denominación del canal, antes TVDoc (Figura 2) y ahora RTVDoc (Figura 3).

Por otra parte, en este mismo periodo de tiempo la existencia de la plataforma originó el establecimiento de un grupo de investigación consolidado complutense con la consiguiente propues- ta de ayuda para la realización de un proyecto de investigación Universidad ComplutenseComunidad Autónoma de Madrid, cuyo valor estratégico y relevancia se concretó en los siguientes puntos (Figura 4).

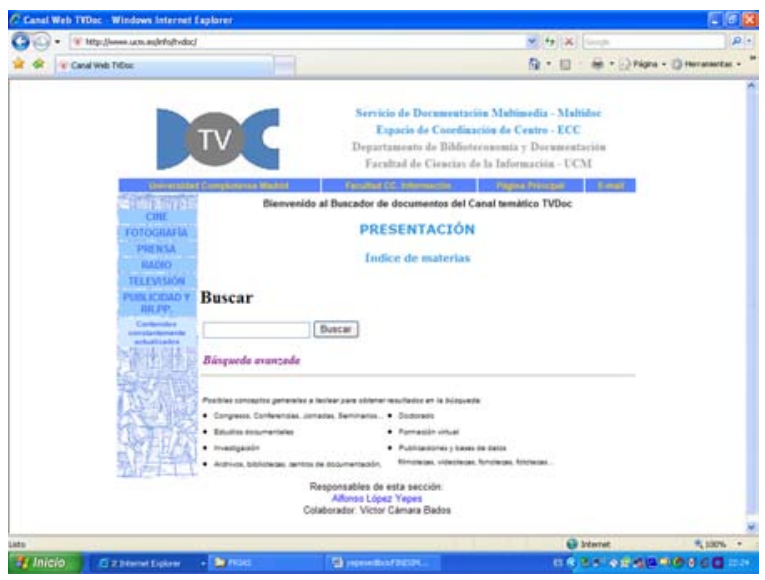

Figura 2

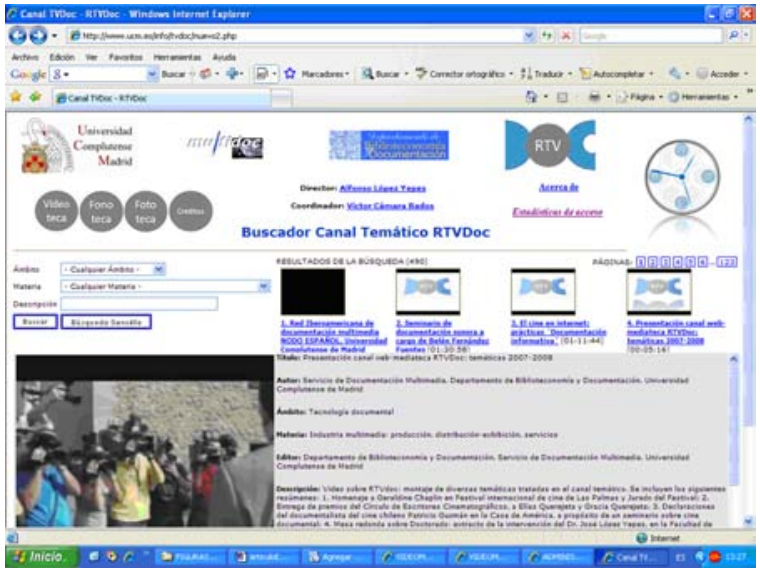

Figura 3

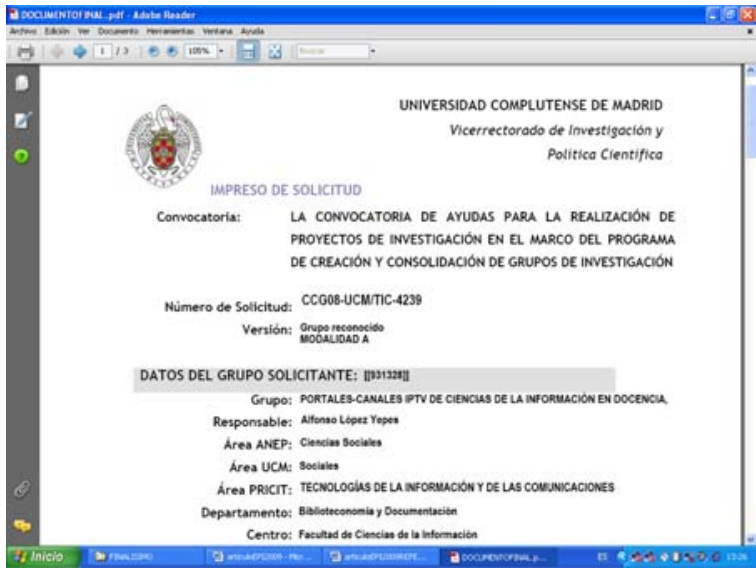

Figura 4 recuperación y difusión en ciencias de la información. // Ibersid. (2009) 169-179. ISSN 1888-0967. 
1. El canal TVDoc vehicula el conocimiento que genera la facultad de ciencias de la información en sus diversos ámbitos en relación con los medios de medios de comunicación.

2. Entre otras funciones, el proyecto de investigación propuesto canaliza entre realizaciones y actuaciones diversas, por ejemplo, los proyectos de investigación y difusión de la producción propia de la facultad de ciencias de la información de Madrid y de sus profesores, alumnos, investigadores y profesionales de los medios de comunicación social, independientemente de la extensión a otras facultades, universidades e instituciones especializadas nacionales e internacionales.

3. La plataforma participa ampliamente de la filosofía que caracteriza a la red de redes como red social basada en el conocimiento distribuido y colaborativo, que es precisamente la filosofía que subyace en la estructura de contenidos que presenta el canal.

El planteamiento y consecución de la investigación contribuirá a la mentalización del uso de la red de redes y el campus virtual de la UCM, puesto que proporciona fondos documentales relevantes en la conformación de asignaturas e investigaciones, tanto presenciales como virtualizadas a través del Espacio de Coordinación de Centro-Facultad de Ciencias de la Información, con el que el canal mantiene una muy estrecha colaboración, pudiendo abrir a su vez diversidad de canales y vías de difusión vía web (más información al respecto en epígrafe Aplicaciones: integración del canal...).

\section{Programación-Mediateca en línea: estructura de contenidos y proceso de trabajo}

El canal temático RTVDoc dispone de una programación permanentemente actualizable como, por ejemplo, emisión en directo de tesis doctorales, seminarios, presentación de publicaciones... y un largo etcétera (véase más adelante el epígrafe "gestión de contenidos multimedia"), actividades estrechamente relacionadas con las ciencias de la información, su estudio e investigación en particular y con la comunicación social en general. Se trata de actividades académicas y profesionales que, una vez emitidas, pasan a engrosar la mediateca del canal para "bajo demanda" ser visionadas en diferido. La tipología de documentos es muy amplia y diversa, como puede apreciarse al acceder al sitio y establecerse una estrategia de búsqueda sobre los contenidos repertoriados mediante la ventana de búsqueda sencilla y avanzada. RTVDoc está accesible en http://www.ucm.es/info/ tvdoc.

A mediados de 2009 la estructura de contenidos y el proceso de trabajo para su sistematización se realizará a través de COMPLUMEDIA (Figura 5), gestor multimedia de la Universidad Complutense, lo que conlleva la integración de RTVDoc en el campus virtual de la UCM y el Taller Multimedia dependiente del departamento de apoyo a la docencia adscrito a los servicios informáticos de la Complutense.

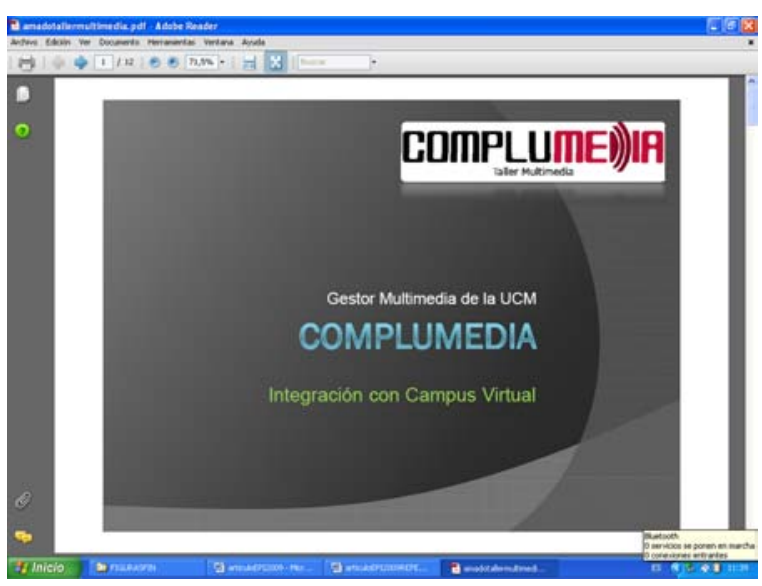

Figura 5

Se trata de un sistema que permite la gestión de los activos multimedia complutenses mediante la inserción, documentación, publicación, preservación, distribución, permisos a usuarios, búsquedas e integración total de contenidos. A propósito de Complumedia y de la integración de contenidos multimedia en el campus virtual de la universidad complutense se celebró en la facultad de filología un taller en el ámbito de la $\mathrm{V}$ Jornada del Campus Virtual de la UCM (Hernández, Pozuelo, López, Cámara, 2009).

\section{Multimedia bajo demanda: texto- audio-imagen fija-vídeo on demand}

La programación genera paralelamente el almacenamiento de la información para ser recuperada posteriormente ("a la carta"), procedente de las distintas tecas que constituyen la mediateca multimedia en línea. La información en ellas contenida es gestionada y descrita estandarizadamente mediante la norma Dublin Core y el gestor de contenidos Videoma (Figura 6), producido y distribuido por la empresa española ISID (http://www.isid.es). Las utilidades del gestor son muy amplias pudiéndose generar contenidos multimedia anejos asociados a los documentos descritos y formatos diversos de vídeo 
digital (mpeg2, wmv, avi) y de otros documentos (HTML, pdf, 16mm., DVD), y de imágenes fijas (jpg, gif, tiff, bmp, png), independientemente de las posibilidades de edición y postproducción en línea -mediante la visualización de keyframes y generación de clips-y sus correspondientes aplicaciones docentes e investigadoras, tanto en el aula como fuera de ella.

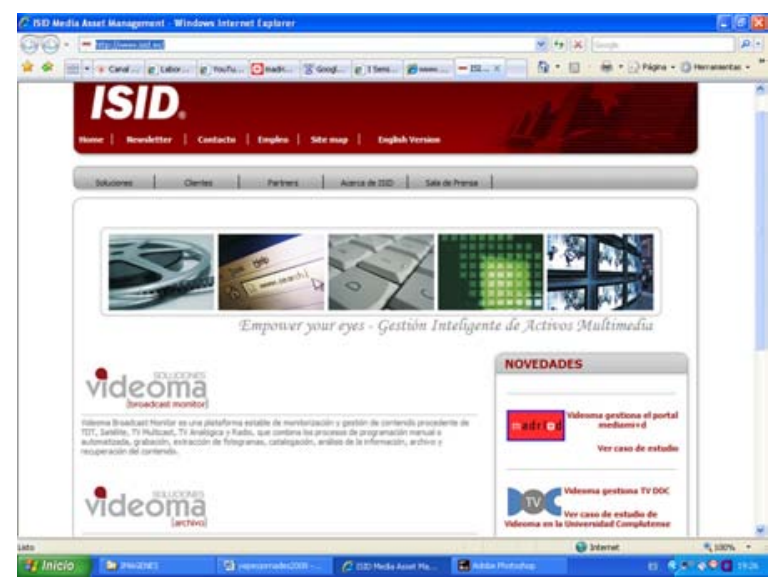

Figura 6

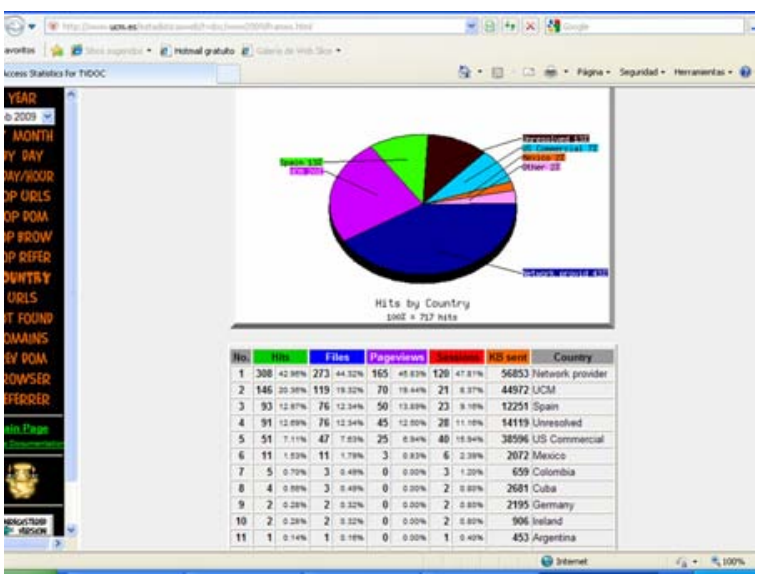

Figura 7

Para facilitar la consulta de los contenidos multimedia del canal se dispone de unas instrucciones de uso, que conviene aquí destacar y que se reproducen a continuación, así como una herramienta que proporciona día a día, mes a mes, año tras año, por países, por dominios, etc. estadísticas de acceso a la plataforma por parte de los usuarios reales (Figura 7):

Los contenidos del canal RTVDoc están en continuo desarrollo y actualización permanentes, así como las materias —en función de la aportación de nuevos contenidos- que describen las temáticas planteadas, a las que se puede acceder mediante búsquedas avanzada (por ámbito, materia y descripción) y sencilla.
La programación en tiempo real vía internet del canal (con reproductor windows media player v. 10 en adelante) se anuncia con la antelación debida, tanto en relación con los propios eventos de la facultad de ciencias de la información de madrid, como en relación con otros en otras instituciones relacionadas, y siempre de interés en el ámbito de las ciencias de la información y de la documentación informativa o de medios de comunicación. Una vez emitidos, los documentos pasan a formar parte de los fondos multimedia de la Mediateca del canal ("multimedia bajo demanda"), para su emisión en diferido siempre que se desee ("a la carta").

El canal se nutre constantemente de documentación originada en fuentes muy diversas y en formatos distintos, procedentes de ámbitos estrechamente relacionados con la documentación de medios informativos.

La información está accesible desde todos los navegadores.

Para la visualización de los contenidos se precisa de una resolución de pantalla de monitor de $1024 \times 680$ (con 800x600 hay problemas).

Para visionar los vídeos se necesita un códec determinado, que si no se detecta automáticamente por no disponerse del mismo, puede bajarse de la red activando el icono indicado para cada sistema operativo en http://www.voiceage.com/acelp_eval _eula.php

\section{Gestión de contenidos multimedia}

La recuperación de los contenidos multimedia del canal (Figura 8), cuyo tratamiento documental sigue basándose en la recuperación normalizada de metadatos en función de trabajos publicados con anterioridad y, más concretamente en la norma Dublin Core (Méndez, 2002, 2006) (Méndez, Bravo, López, 2007) (López, Pérez, Sánchez, 2003) (Pérez, López, Sánchez, 2003) se estructura de momento en tres campos fundamentales: ámbito, materia y descripción.

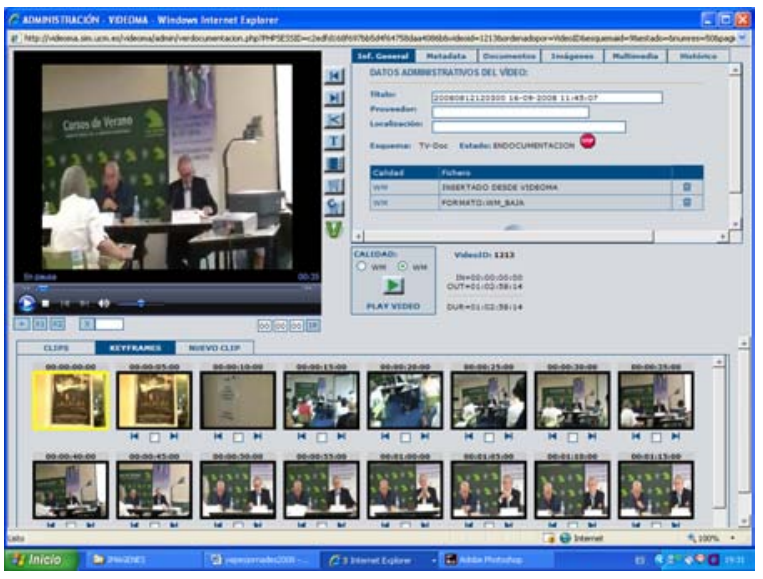

Figura 8 recuperación y difusión en ciencias de la información. // Ibersid. (2009) 169-179. ISSN 1888-0967. 


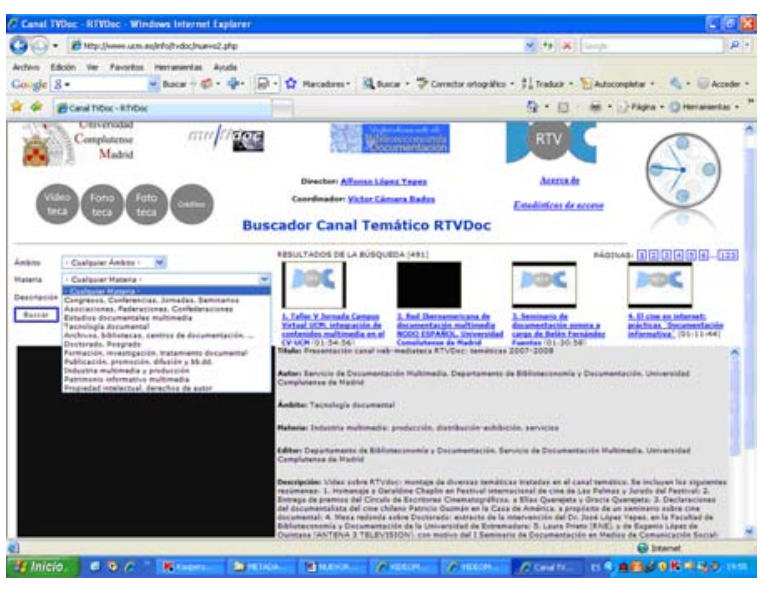

Figura 9

El primero contempla siete ámbitos de estudio e investigación: cine, fotografía, prensa, radio, televisión, publicidad-relaciones públicas y tecnología documental, que corresponden a los estudios e investigación en ciencias de la información. Y el campo de materia se despliega en once líneas temáticas que afectan en todo momento a dichos ámbitos y que se desdoblan en submaterias susceptibles permanentemente de un desarrollo temático más pormenorizado, como por ejemplo el relacionado a continuación (Figura 9):

- Congresos, conferencias, jornadas, seminarios, talleres...

- Asociaciones, Federaciones, Confederaciones...

- Estudios documentales multimedia: dosieres informativos, investigaciones de curso, estudios monográficos, trabajos de investigación...

- Tecnología documental: sistemas documentales hardware-software, sistemas de almacenamiento, producción, recuperación y difusión, redes (listas, foros, portales, comunidades virtuales, news, herramientas de intercomunicación, sistemas de transmisión, streaming...).

- Archivos, bibliotecas, centros de documentación, filmotecas, videotecas, fonotecas, fototecas, mediatecas.

- Doctorado, posgrado, títulos propios: trabajos de investigación-DEA, defensa de tesis doctorales, mesas redondas.

- Formación, investigación, tratamiento documental: formación semipresencial, formación virtual, presentación-exposición de investigaciones.
- Publicación, promoción y difusión: fuentes de información, publicaciones, bases de datos; medios de comunicación impresos, audiovisuales y multimedia; festivales, ferias, premios...; presentación de publicaciones...; editoriales, librerías especializadas...

- Industria multimedia: producción, distribuciónexhibición, servicios; preproducción, postproducción...; pases de prensa, pre-estrenos, estrenos...; rodajes.

- Patrimonio informativo multimedia: filmotecas.

- Propiedad intelectual, derechos de autor.

\section{Mediateca-Fonoteca, fototeca, hemeroteca y videoteca: documentación informativa (cine, fotografía, prensa, publicidad-relaciones públicas, radio, televisión, tecnología documental)}

Precisamente porque RTVDoc supone la programación en tiempo real, previa comunicación del día, hora y lugar de emisión del evento, también ello conlleva el almacenamiento simultáneo en forma de documento, para su recuperación posterior, en diferido, de la actuación programada. Ello supone la funcionalidad añadida de mediateca en línea y, en consecuencia, la labor que desarrolla el canal web como servicio de información y documentación en línea suministrador de numerosos y diversos materiales didácticos multimedia, como en el epígrafe inmediatamente anterior se daba cumplida cuenta de ello. Las temáticas tratadas, en permanente estado de actualización y ampliación, presentan un interés evidente para docentes, estudiantes, investigadores, comunicadores y profesionales de la información y documentación, recuperables a través de los tres campos de consulta ya mencionados con contenidos estandarizados en forma de metadatos.

Una muestra de lo recién expresado presenta la página de bienvenida del canal web-mediateca, en su arranque, en forma de aspectos diversos sobre documentación informativa en torno a la comunicación periodística, audiovisual y publicitaria y de relaciones públicas, abordadas en todo momento con destino al canal. Para que el lector de estas páginas se haga idea de algunos, solamente algunos, de los contenidos programados, valgan los siguientes ejemplos: Homenaje a Geraldine Chaplin en Festival internacional de cine de Las Palmas y Jurado del Festival; Entrega de premios del Círculo de Escritores Cinematográficos a Elías Querejeta y Gracia Querejeta; Declaraciones del documentalista del cine chileno Patricio Guzmán en la Casa de América, a propósito de un seminario 
sobre cine documental; Mesa redonda sobre estudios de doctorado en la universidad española: extracto de la intervención del Dr. José López Yepes, en la Facultad de Biblioteconomía y Documentación de la Universidad de Extremadura; Declaraciones de Laura Prieto, documentalista de RNE, y de Eugenio López de Quintana, subdirector del centro de documentación de ANTENA 3 TELEVISION, con motivo del I Seminario de Documentación en Medios de Comunicación Social: Ciberperiodismo y Ciberdocumentación, en la Facultad de Ciencias de la Información de la Universidad Computense de Madrid; Intervención del Prof. Joaquin Aguirre, con motivo de "Diálogos Complutense" en la facultad de ciencias de la información de Madrid; Declaraciones del profesor Juan Marcos Recio durante el mencionado I Seminario de Documentación en Medios de Comunicación Social: Ciberperiodismo y Ciberdocumentación.

$Y$ otros tantos temas.... muy variados como, incluso, investigaciones realizadas en el ámbito teórico-práctico de la disciplina Documentación Informativa, que se imparte en las tres secciones de periodismo, comunicación audiovisual y publicidad y relaciones públicas de los estudios de ciencias de la información (Campo, Camps, 2008) incluida la emisión de programas de radio y su almacenamiento en la Fonoteca para su posterior difusión en diferido "a la carta". La programación radiofónica se emite en tiempo real, previo aviso y difusión informativa en cuanto a día y hora, a través de 10http://www.ucm. es/info/tvdoc/radiodoc.php (Figura 10).

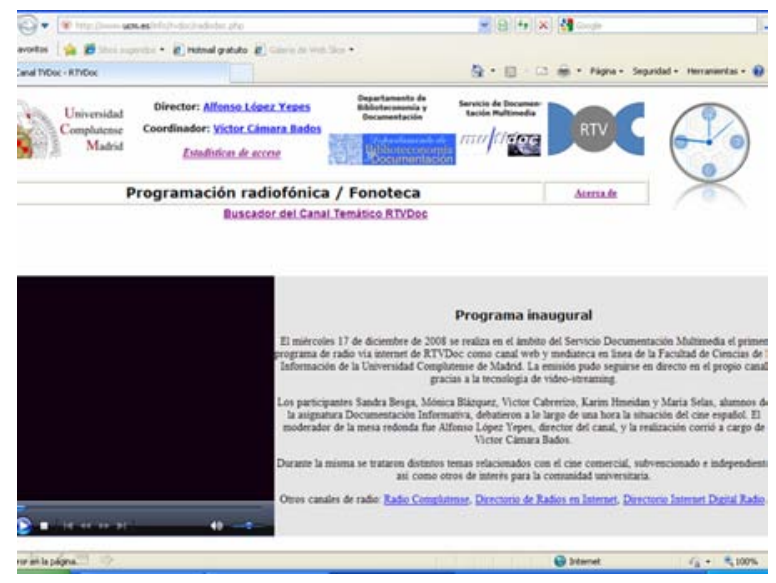

Figura 10

A ello se añade, en fin, el acceso a un corpus de materiales de interés para la asignatura mencionada, como producto de varios proyectos de innovación educativa financiados por el vicerrectorado de investigación de la universidad complutense, entre los que se incluye una mediate- ca de documentación cinematográfica en línea. Para mayor abundamiento en cuanto a materiales de trabajo con destino a la investigación cinematográfica, el acceso al fondo documental digitalizado del cineasta José Val del Omar, depositado en la Filmoteca de Andalucía en Córdoba, como consecuencia del proyecto financiado por la la Junta de Andalucía con la colaboración del Servicio de Documentación Multimedia de la Universidad Complutense. Existe abundante información sobre los proyectos de investigación subvencionados en los siguientes tres sitios:

\section{- http://multidoc.rediris.es/departamento}

- http://multidoc.rediris.es/mediateca

- http://multidoc.rediris.es/valdelomar

En fin, las estadísticas de contenidos de la mediateca ascienden, en el momento de elaboración y cierre (20 de febrero de 2009) de esta colaboración con destino al Profesional de la Información, vol. 18, núm. 3, mayo-junio de 2009, a unos 500 vídeos, en torno a 1.500 fotografías que conforman la Fototeca (Figura 11), algunos documentos sonoros (en número todavía escaso) y a numerosos enlaces a recursos hemerográficos en forma de directorio temático, que constituyen la Hemeroteca del canal.
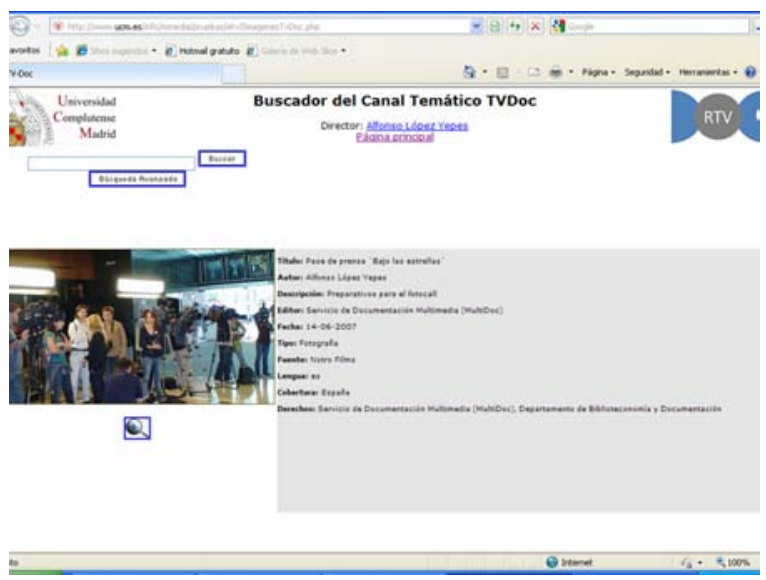

Figura 11

\section{Aplicaciones: integración del canal en ámbitos de estudio e investigación en documentación informativa}

En función de lo expresado en epígrafes previos, RTVDoc cumple también como servicio de información y documentación integrándose en ámbitos de estudio e investigación relacionados con las ciencias de la información y la documentación informativa. Algunos ejemplos de ello, materializados en documentos depositados en la mediateca del canal se comentan a continua- 
ción. En cuanto al primer y segundo ciclo de estudio de la disciplina Documentación informativa, en su programa teórico-práctico, se dispone de presentaciones-exposiciones de alumnos en el aula, basadas en metodologías expresadas en clase en cuanto a sistematización de contenidos, en relación con las siguientes temáticas: redes sociales, buscadores, canales IPTV, cine en internet, edición de sonido, diseño de páginas web, etc. (Blázquez, Díaz, 2008) (Figura 12).

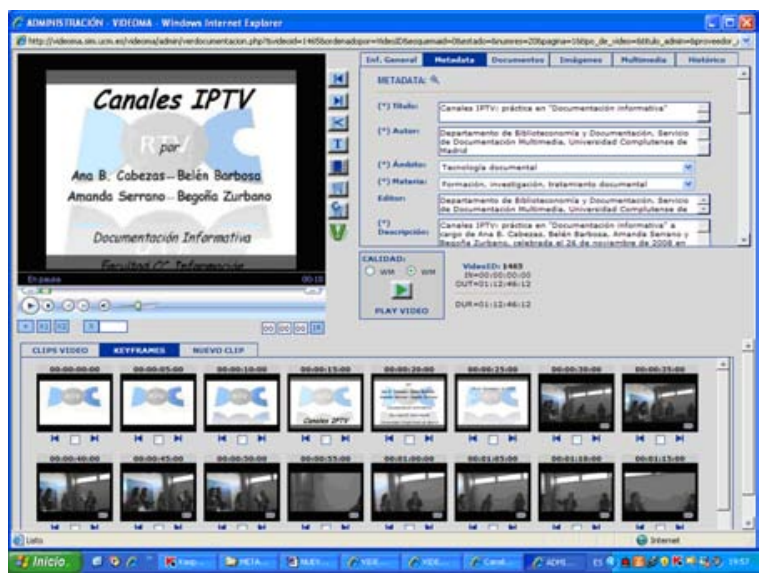

Figura 12

En tercer ciclo-doctorado también está presente la mediateca del canal como herramienta de recuperación y visionado de contenidos, así como en postgrado, más concretamente en el máster de comunicación social, el máster de periodismo y el máster en gestión de la documentación y bibliotecas (Figura 13).

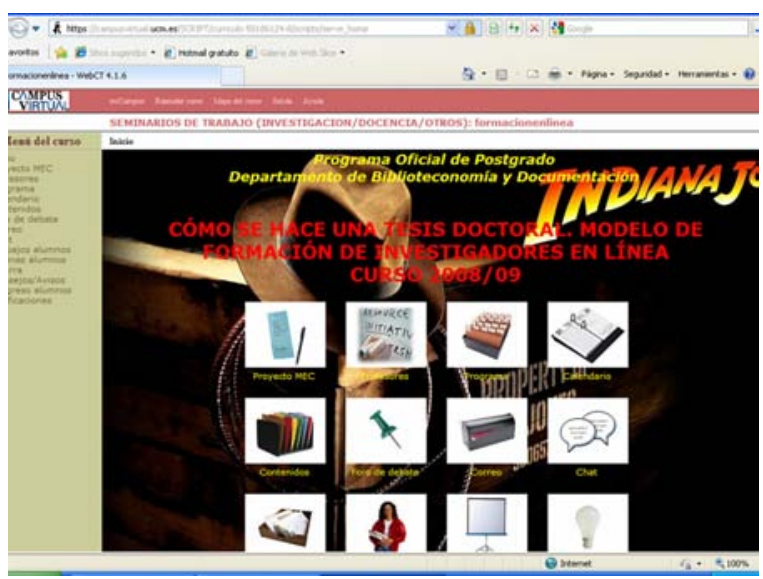

Figura 13

En sus programas lectivos se contempla, respectivamente, la documentación informativa en forma de "Practicum: Taller de documentación aplicada", "Documentación en contenidos digita- les" y "Documentación audiovisual y multimedia". También se hace uso del canal web en el marco de los títulos propios de la universidad complutense, a propósito del título "Experto en Información y Documentación Ambiental". Incluso, en fin, es materia de estudio y aplicación en el marco de máster de otras universidades como es el caso del Máster Online en Documentación Digital de la Universitat Pompeu Fabra (López, 2008a).

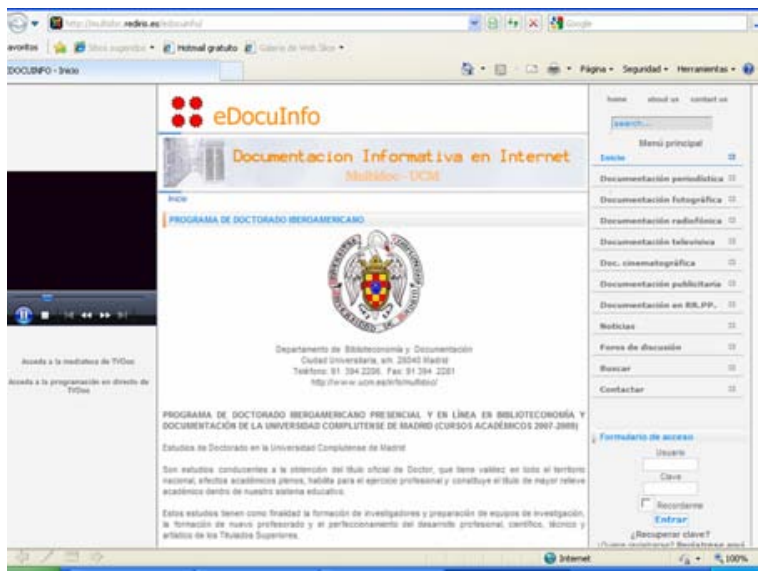

Figura 14

Igualmente, la utilización del canal con todas sus funcionalidades y posibilidades está presente en otros ámbitos específicos involucrados en la gestión de la información en relación con los medios de comunicación social, como son el portal de portales de documentación informativa e-Docuinfo (Figura 14), el Espacio de Coordinación de Centro (ECC) para el acceso al campus virtual de la UCM (Figura 15).

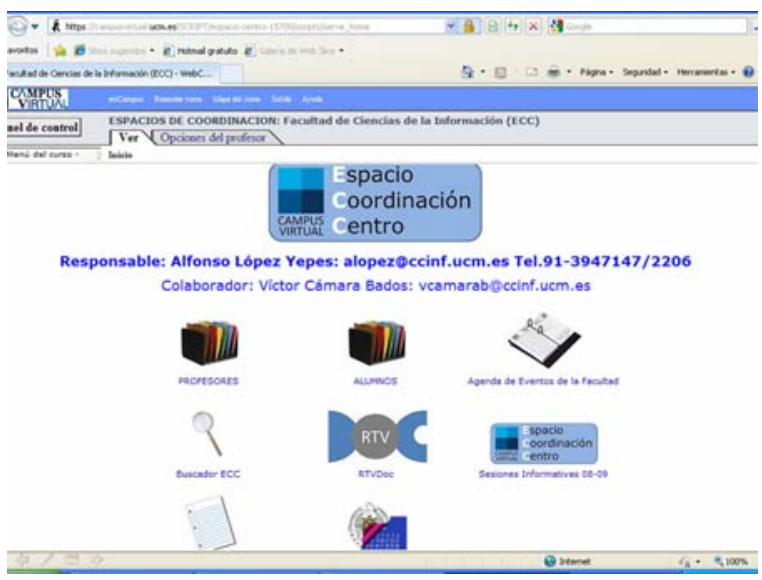

Figura 15

o la propia página de bienvenida de la facultad de ciencias de la información de madrid en la que el canal aparece como un servicio de infor- 
mación destinado tanto a los casi 6.500 alumnos y cerca de 350 profesores, como a docentes, discentes e investigadores de otras universidades y profesionales especializados.

\subsection{Universidad-Empresa informativa:}

\section{colaboraciones}

La presencia del canal temático se extiende a otras instituciones y empresas informativas, con proyectos en la misma o parecida línea, contribuyendo así a un enriquecimiento progresivo de la influencia de este canal temático en el ámbito docente y de investigación, favoreciendo así la colaboración universidad-empresa informativa. En estos momentos, entre otras colaboraciones, por lo que conlleva de producción propia de materiales didácticos multimedia y la consiguiente distribución del conocimiento científico, conviene destacar la colaboración que se ha hecho extensible a empresas informativas del calado de Cineinforme y Teleinforme (a propósito de Cine y Tele On Line), con una presencia importante de RTVDoc (Figura 16).

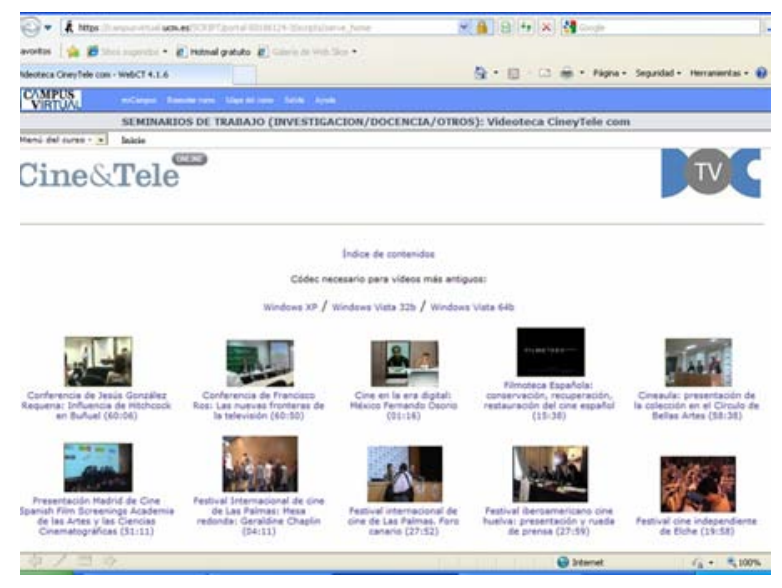

Figura 16

Asimismo, la colaboración - vía convenio- con la Facultad de Biblioteconomia y Documentación de la Universidad de Extremadura, a propósito de Onda Campus (Figura 17) y el intercambio colaborativo y distributivo de documentación radiofónica y televisiva. $\mathrm{O}$ el acuerdo de colaboración por parte de Baratz-Servicios de Teledocumentación materializado en la financiación, en parte, de la puesta en marcha del Laboratorio multimedia de formación en línea dependiente del Servicio de Documentación Multimedia-Multidoc (Figura 18).

A resaltar, finalmente, la petición por parte de la Asociación Española de Análisis Transaccional (AESPAT) de la participación de RTVDoc en la programación y difusión de contenidos del XIV
Congreso Español de Análisis Transaccional, que transcurrió en la facultad de ciencias de la información de madrid durante los días 6,7 y 8 de marzo de 2008 (Figura 19).

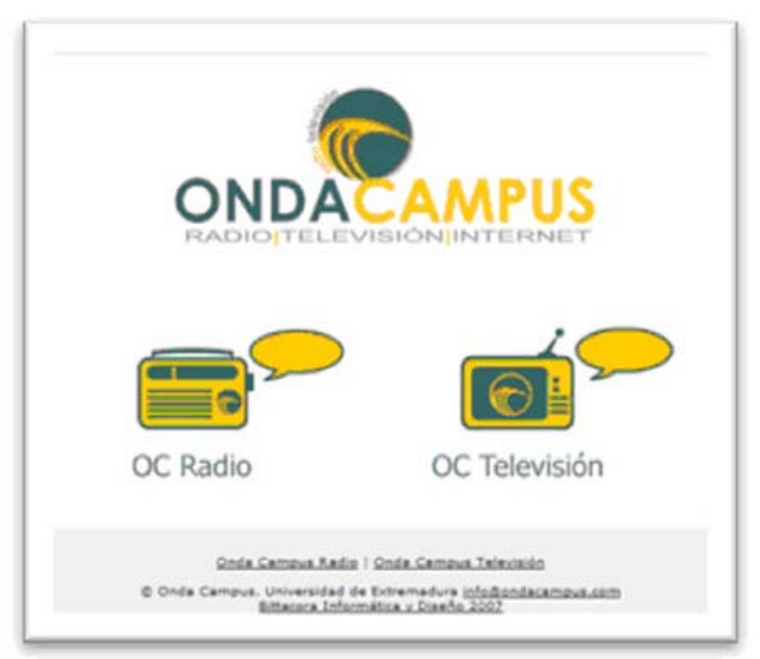

Figura 17

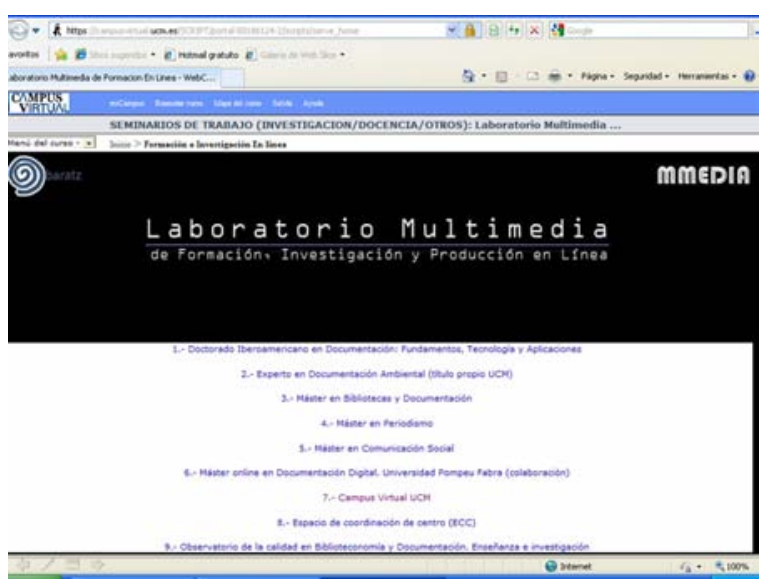

Figura 18

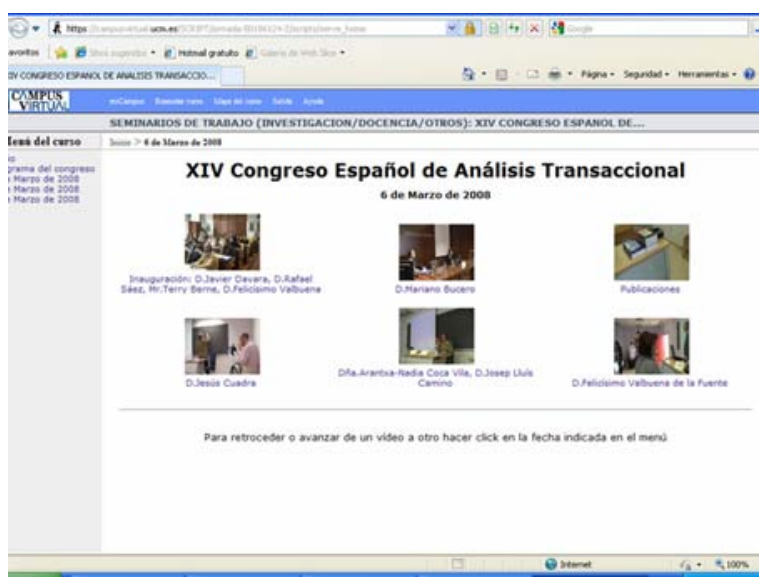

Figura 19 recuperación y difusión en ciencias de la información. // Ibersid. (2009) 169-179. ISSN 1888-0967. 


\section{Difusión informativa de las actuaciones de RTVDoc}

Este artículo destaca en todo momento las labores de producción de contenidos y recuperación de los mismos que se está llevando a cabo en el marco de RTVDoc, pero también debe destacarse que la producción informativa es importante y necesaria, pero no lo es menos la difusión informativa que se haga de ella y de la transmisión de conocimiento que ello supone. Por esta razón, se procede a continuación a mencionar los ámbitos de difusión que ha protagonizado el canal durante los dos últimos años.

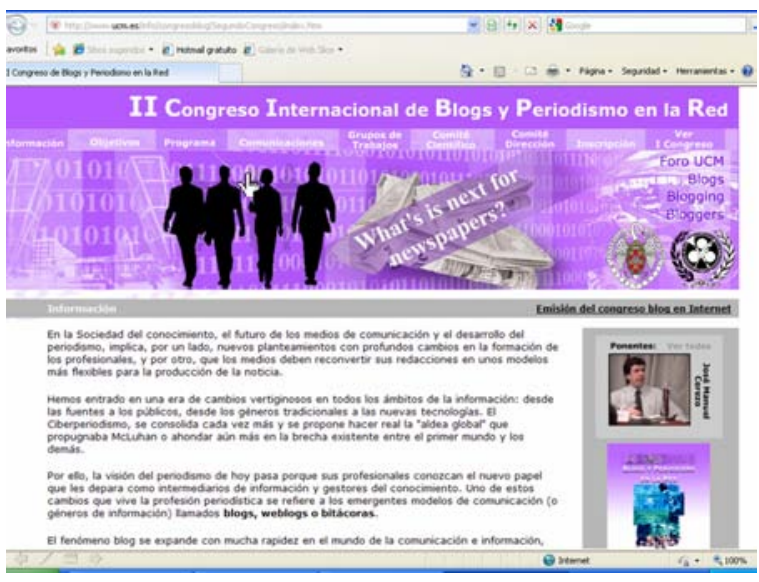

Figura 20

En los dos años previos de existencia del canal web, 2007 y 2008, la difusión informativa del mismo se ha realizado en destacados ámbitos de la comunicación social y , más concretamente, de la documentación informativa. En 2007 estuvo presente -en su fase inicial- en esta misma publicación (López, Sánchez, 2007) y en ámbitos profesionales como congresos y seminarios: en el II Congreso de Blogs y Periodismo en Red (II Congreso Internacional de Blogs y Periodismo en la Red http://www.ucm.es/ info/congresoblog/SegundoCongreso/index.htm (Figura 20) y en el I Seminario de Documentación en los medios de comunicación: ciberperiodismo y ciberdocumentación (http://www.ucm. es/info/ciberdocumentacion/ (Figura 21).

En el transcurso de 2008 fue todavía mayor la difusión informativa de RTVDoc (todavía entonces TVDoc), en foros profesionales nacionales e internacionales, como en el marco del Congreso fundacional I+C Investigar la Comunicación (AEIC), organizado por la Asociación Española de Investigación en Comunicación en la Facultad de Ciencias de la Comunicación de la Universidad de Santiago de Compostela durante los días 30 de enero a 1 de febrero (López, 2008b).
O también en Salamanca, el 7 de febrero en las instalaciones de la facultad de traducción y documentación de la universidad de Salamanca, con motivo del III Encuentro Ibérico de Docentes e Investigadores en Información y Documentación EDIBCIC 2008 (López, 2008c). Y, finalmente, en el ámbito nacional, en el marco de las 11 Jornadas de Información y Documentación de Barcelona (López, Cámara, 2008) (Figura 22).

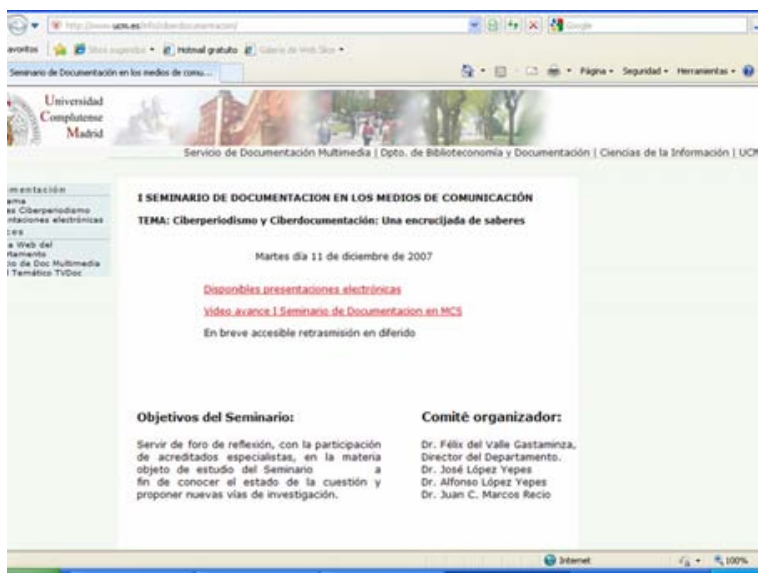

Figura 21

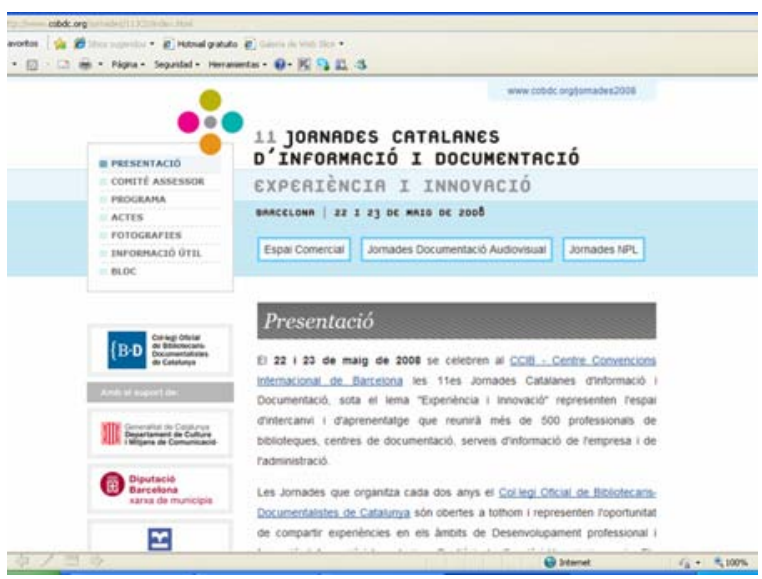

Figura 22

En cuanto a foros internacionales, concretamente latinoamericanos, la presencia del canal se hizo realidad, sucesivamente, en Perú, México y Cuba, a propósito respectivamente del I Seminario hispano-peruano de Bibliotecología y Documentación, del V Seminario hispanomexicano de Investigación en Bibliotecología y del Congreso Internacional de Información INFO 2008. En los dos primeros casos, en cuya propuesta de organización intervino directamente el departamento de biblioteconomía y documentación de la universidad complutense, participaron el Centro Universitario de Investigaciones Bibliotecológicas (CUIB) de la Universidad Nacional 
Autónoma de México (UNAM) y la Biblioteca Nacional del Perú (BNP), la Universidad Nacional Mayor de San Marcos y la Pontificia Universidad Católica del Perú. En el tercer caso, INFO 2008, el Congreso Internacional de Información está dando cobertura informativa del canal web a través de su página de bienvenida en internet, con motivo de la ponencia presentada por uno de los autores de este trabajo (López, Prat, 2008) (Figura 23).

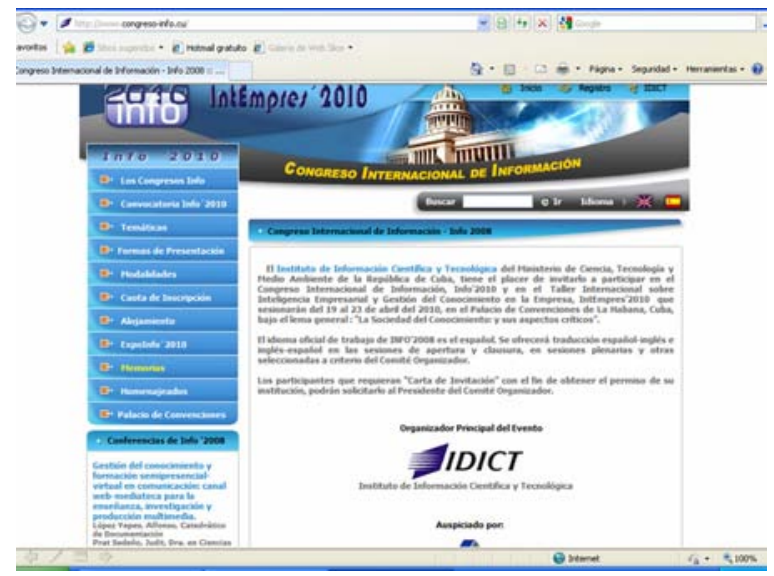

Figura 23

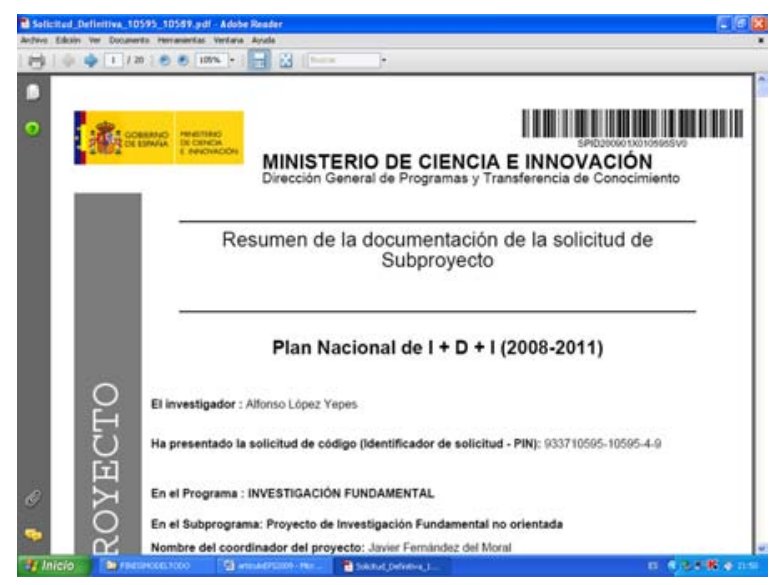

Figura 24

Finalmente, en cuanto a las perspectivas futuras de RTVDoc debe señalarse que éste se orienta hacia su actualización permanente de contenidos y ámbitos de aplicación, así como su consolidación y difusión todavía mayores a través del Plan Nacional de I+D+I convocado por el Ministerio de Ciencia e Innovación (2008-2011). El canal web-mediateca en línea participa y está implicado en el proyecto conjunto Nuevos fundamentos y aplicaciones de la comunicación social en la era de la globalización, integrado en forma de subproyecto bajo el título "Gestión de la información digital en la era de la globaliza- ción: portales, canales temáticos web y mediatecas en línea para la investigación y producción multimedia en comunicación social" (Figura 24).

\section{Conclusiones}

Este trabajo apuesta por la consolidación definitiva del canal temático RTVDoc plataforma web para la formación, investigación, recuperación y difusión de materiales didácticos multimedia en el ámbito de las ciencias de la información; y más concretamente de los archivos, servicios y centros de documentación de medios de comunicación social o medios informativos. Se destacan asimismo las aplicaciones de los contenidos del canal en internet en el área de la formación semipresencial y virtual aplicada al estudio de las ciencias informativas. Este trabajo, en fin, concluye con los siguientes puntos:

1. Se consolida el establecimiento de canales WebRTV en el marco de la web social, y más concretamente, en nuestro caso, en el ámbito de la documentación informativa, con la puesta en marcha definitiva del canal webmediateca RTVDoc.

2. La actualización permanente y la proyección colaborativa y distribuida de sus contenidos, así como la difusión de los mismos a nivel ciberespacial.

3. La plataforma propuesta supone una programación permanente en tiempo real y en diferido, en forma de mediateca: hemeroteca, fonoteca, fototeca, videoteca.

4. El concepto "vídeo bajo demanda" ha evolucionado, en consecuencia, hacia el establecimiento de un nuevo concepto: "multimedia bajo demanda".

\section{Notas}

(*) Alfonso López Yepes es catedrático de documentación, director del Servicio de Documentación Multimedia de la Universidad Complutense y director de la revista Cuadernos de documentación multimedia. Coordina el Doctorado en línea "Documentación: fundamentos, tecnología y aplicaciones (2007-2009)". Responsable en la facultad de ciencias de la información de Madrid del Espacio de Coordinación de Centro para el acceso al campus virtual de la UCM.

$\left.{ }^{* *}\right)$ Víctor Cámara Bados es máster en producción audiovisual, colaborador del Servicio de Documentación Multimedia de la Universidad Complutense y del Espacio de Coordinación de Centro para el acceso al campus virtual de la UCM, en cuyo diseño, asesoramiento y producción de contenidos participa. Coordinador del canal webmediateca RTVDoc 


\section{Referencias}

Blázquez, Mónica y Díaz, Ester (2008). Buscadores. Madrid, Servicio de Documentación Multimedia-RTVDoc, Facultad de Ciencias de la Información, Universidad Complutense, 17 de diciembre de 2008, 41 minutos (vídeo formato .wmv)

http://videoma.sim.ucm.es/videoma/wm/wmv_baja_yepes/32 0476/147 961146 videos 1463 WM BAJA.wmv (2009-05-09)

Cabezas, Ana B.; Barbosa, Belén; Serrano, Amanda y Zurbano, Begoña (2008). Canales IPTV. Madrid, Servicio de Documentación Multimedia-RTVDoc, Facultad de Ciencias de la Información, Universidad Complutense, 26 de noviembre de 2008, 73 minutos (vídeo formato .wmv). http://videoma.sim.ucm.es/videoma/wm/wmv ba ja_yepes/320476/147_96_1_146_videos_1465_WM_BA JA.wmv

Campo de Luna, Laura y Camps Irene (2008). Redes sociales. Madrid, Servicio de Documentación MultimediaRTVDoc, Facultad de Ciencias de la Información, Universidad Complutense, 3 de diciembre de 2008, 66 minutos (vídeo formato .wmv). http://videoma.sim.ucm. es/videoma/wm/wmv baja yepes/320476/147 96_1_14 6_videos_1369_WM_BAJA.wmv (2009-05-09).

Hernández Pascual, Aurelio; Pozuelo Sanz, Amado José; López Yepes, Alfonso y Cámara Bados, Víctor (2009). A propósito de Complumedia. Integración de contenidos multimedia en el campus virtual. V Jornada Campus Virtual UCM, Taller. RTVDoc. Madrid, Servicio de Documentación Multimedia, Facultad de Ciencias de la Información, Universidad Complutense, 10 de febrero de 2009, 115 minutos (vídeo formato .wmv) http://videoma.sim.ucm.es/videoma/wm/01_WM_Baja_U CM_General/147_96_1_146_videos_1498_WM_BAJĀ. wmv (2009-05-09).

Jiménez, Irene (2008). Especial web tv. La convergencia de Televisión e Internet: la Web TV. Teleinforme, diciembre 2008, págs.23-31.

López Yepes, Alfonso (2008a). Documentación cinematográfica: el cine en la era digital [en línea]. // Rovira, C.; Lluís Codina (dir.) Documentación digital. Barcelona: Grupo de Investigación DigiDoc. Instituto Universitario de Lingüística Aplicada. Departamento de Periodismo y de Comunicación Audiovisual. Universidad Pompeu Fabra, 2008 http://www.documentaciondigital.org (2009-0509).

López Yepes, Alfonso (2008b). TVDoc: un canal temático de documentación informativa en Internet. Actas del Congreso I+C Investigar la Comunicación (AE-IC). Barcelona, Asociación Española de Investigación en Comunicación, 2008 (CD-ROM). http://www.ae-ic.org/esp/ home.asp (2009-05-09).

López Yepes, Alfonso (2008c). Campus virtual y mediateca en línea: una experiencia docente innovadora en el ámbito de la documentación informativa. // Actas del III Encuentro Ibérico de Docentes e Investigadores en Información y Documentación EDIBCIC 2008. Salamanca, EDIBCIC, 2008.

López Yepes, Alfonso y Cámara Bados, Víctor (2008). Documentación cinematográfica en línea y en diferido: canal web TVDoc. // Cuadernos de Documentación Multimedia.19 (2008). http://multidoc.rediris.es/cdm (200905-09).

López Yepes, Alfonso; Pérez Agüera, José Ramón y Sánchez Jiménez, Rodrigo (2003). Tratamiento de la documentación audiovisual en el entorno digital: iniciativas de metadatos y lenguajes de descripción multimedia. // El Profesional de la Información.12:6 (noviembrediciembre 2003) 443-451. http://www.elprofesional delainformacion.com/contenidos/2003/noviembre/3.pdf (2009-05-09).

López Yepes, Alfonso y Prat Sedeño, Judit (2008). Gestión del conocimiento y formación semipresencial-virtual en comunicación. // Actas del Congreso Internacional de Información INFO2008, abril 2008. La Habana (Cuba), INFO2008, 2008 (CD-ROM) http://www.congreso-info.cu/

López Yepes, Alfonso; Sánchez Jiménez, Rodrigo (2007). eDoculnfo/TVDoc: portal de documentación informativacanal temático IPTV. // El Profesional de la Información, Vol. 16, núm.5, septiembre-octubre 2007, págs.456-467. http://elprofesionaldelainformacion.metapress.com/app/h ome/contribution.asp?referrer=parent\&backto=issue $, 8,1$ 8;journal,9,63; homemainpublications, 1,1;

Méndez Rodríguez, Eva María (2002). Metadatos y Recuperación de información: estándares, problemas y aplicabilidad en Bibliotecas Digitales. Gijón: Trea, 2002. 419 p.

Méndez Rodríguez, Eva María (2006). Dublin Core, metadatos y vocabularios. // El Profesional de la Información. 15:2 (marzo-abril 2006) 84-86. http://elprofesional delainformacion.metapress.com/app/home/contribution .asp?referrer=parent\&backto=issue, 1,9;journal,8,53; hom emainpublications, 1,1 (2009-05-09).

Méndez Rodríguez, Eva; Bravo, Alejandro y López, Leandro Mariano (2007). Microformatos: web 2.0 para el Dublin Core. // El Profesional de la Información, Vol.16, núm.2, marzo-abril 2007. http://elprofesionaldelainformacion .metapress.com/app/home/contribution.asp?referrer=par ent\&backto=issue ,3,15;journal, 12,63; linkingpublicationre sults, 1:105302,1 (2009-05-09).

Pérez Agüera, José Ramón; López Yepes, Alfonso; Sánchez Jiménez, Rodrigo (2003). Un modelo para el diseño de sistemas dinámicos de gestión de información multimedia. // Travieso Rodríguez, Críspulo y Frías Montoya, José Antonio (Coords.). Tendencias de investigación en organización del conocimiento. Salamanca, Facultad de Documentación, Universidad de Salamanca, 2003. 645-650.

Delclaux, Isidoro; Seoane, Julio (1982). Psicología cognitiva y procesamiento de la información: teoría, investigación y aplicaciones. Madrid: Ediciones Pirámide, 1982.

Ellis, David (1992a). The physical and cognitive paradigms in Information Retrieval Research. // Journal of Documentation. 48:1 (March 1992) 45-46.

Markey, Karen (1990). Keyword searching in an online catalog enhanced with a library classification. // Bengtson, Betty G.; Hill, Janet Swan (eds.). Classification of library materials: current and future potential for providing access. New York: Neal-Shuman Publishers, 1990. 99125.

Sagredo Fernández, Félix; Espinosa Temiño, María Blanca (2000). Del libro, al libro electrónico-digital. // Cuadernos de Documentación Multimedia. 9 (2000).

Smith, Ph. J.; Beghtol, C.; Fidel, R.; Kwasnik, B. H. (eds.) (1993). Proceedings of the 4th ASIS SIG/CR Classification Research Workshop: Columbus, OH, Oct.24, 1993. Silver Spring, MD.: American Society for Information Science, 1993 